Planetary Systems in the Universe - Observation, Formation and Evolution

Proceedings IAU Symposium No. 202, (C)2004 IAU

Alan Penny, Pawel Artymowicz, Anne-Marie Lagrange, 6 Sara Russell, eds.

\title{
Evidences of protoplanetary disks in a selected sample of weak T Tauri stars
}

\author{
Jane Gregorio-Hetem \\ Universidade de São Paulo (IAG/USP), Av. Miguel Stéfano 4200, \\ 04301-904, São Paulo - Brazil; jane@orion.iagusp.usp.br
}

Annibal Hetem Jr.

Universidade Paulista (UNIP/ICET), R. Dr. Bacelar 1212, 04026-002, São Paulo - Brazil; annibal.hetem@usa.net

\begin{abstract}
A model with two dust components is used do explain the circumstellar structure of weak-T Tauri stars. The IR-excess was calculated and compared to spectroscopic criteria in order to classify the objects according an evolutionary sequence. About $46 \%$ of the sample correspond to young main sequence stars showing dust distribution consistent with a disrupted disk, that could be possibly caused by the formation of a planetary system.
\end{abstract}

\section{Introduction}

It has been increasing the interest in better classifying the evolutionary status of T Tauri (TTs) recently discovered by the X-rays surveys. The significant number of weak-TTs candidates being confirmed as post-TTs or young main sequence (YMS) objects, unless pre-main sequence (PMS) low-mass stars (e.g. Briceño et al. 1997), has motivated several works to investigate the nature of these stars. By considering that a fraction of these objects could have a $\beta$ Picor Vega-like circumstellar structure, the analysis of larger samples of weak-TT could provide more candidates showing evidences of protoplanetary disks. This fact leads to a more exciting aspect in better classifying PMS stars.

A search for young stellar objects based on IRAS colours, Pico dos Dias Survey (Gregorio-Hetem et al. 1992 - PDS I; Torres et al. 1995 - PDS II), revealed almost a hundred of austral TTs. In order to verify the occurrence of post-TTs or YMS objects among the stars listed by PDS I and PDS II, we selected a sample of 26 TTs having: E.W. $(\mathrm{H} \alpha)<10 A$, available spectral type, and $U B V R I$ and IRAS data. The main goal of this study is to compare the classification obtained from spectroscopic criteria with other indicators of the dust distribution in the circumstellar structure. The present work describes the analysis of the sample and shows the results obtained for the objects identified as YMS. 
Table 1. List of the stars classified as young main sequence objects.

\begin{tabular}{lccrrrrr}
\hline Object & $T_{\text {eff }}$ & $W_{L i}(\AA)$ & $L_{d} \%$ & $L_{e} \%$ & {$[12-25]$} & {$[25-60]$} & $a_{I R}$ \\
\hline PDS030 & 6390 & 0.13 & 19.3 & 11.2 & 2.44 & 1.57 & -0.5 \\
PDS035 & 6252 & 0.13 & 18.7 & 35.3 & 2.56 & 2.48 & -0.4 \\
PDS093 & 6653 & 0.01 & 16.2 & 4.5 & 2.28 & 2.06 & -1.4 \\
PDS109 & 6390 & 0.11 & 3.4 & 10.6 & 2.03 & 1.89 & -1.2 \\
PDS110 & 6653 & 0.08 & 13.6 & 8.7 & 2.75 & 2.14 & -1.5 \\
PDS111 & 5715 & 0.23 & 11.3 & 5.2 & 2.77 & 2.29 & -1.8 \\
PDS115 & 5636 & 0.13 & 21.5 & 0.3 & 2.09 & 1.50 & -1.4 \\
PDS116 & 5636 & 0.26 & 17.3 & 0.8 & 1.75 & 1.71 & -1.4 \\
PDS118 & 6531 & 0.06 & 17.7 & 1.6 & 1.83 & 1.69 & -1.4 \\
PDS119 & 6653 & 0.15 & 0.0 & 22.2 & 2.81 & 1.23 & -2.6 \\
PDS145 & 4446 & 0.20 & 27.0 & 33.6 & 1.97 & 1.56 & -0.1 \\
PDS156 & 5943 & 0.09 & 26.8 & 19.6 & 1.92 & 1.29 & -0.3 \\
\hline
\end{tabular}

\section{Analysis}

The classification of PMS low-mass stars using spectroscopic criteria proposed by Martín (1997) was adopted. The measured equivalent width of the lithium line is plotted as a function of $T_{\text {eff }}$ in a diagram where different locations for weak-TT, post-TT and YMS stars are indicated. Table 1 lists twelve stars of the sample, corresponding to the YMS category. The results were confirmed by using other similar criteria based on the Li- $T_{\text {eff }}$ correlation (Covino et al. 1997; Wichmann et al. 1999). All of the objects show infrared excess, evaluated by means of the near-IR spectral index and the IRAS colours. A model to fit the spectral energy distribution (SED) was applied in order to estimate the individual contribution in the emitted energy from different dust components.

\subsection{Infrared Excess}

In order to evaluate the SED slope in the $2-10 \mu \mathrm{m}$ range, we estimated the spectral index $a_{I R}=d\left(\log \left(\lambda F_{\lambda}\right)\right) / d(\log \lambda)$, proposed by Wilking, Lada \& Young (1989) and revised by André \& Montmerle (1994). In this case, the stars showing $a_{I R}<-1.5$ are considered as Class III objects, which corresponds to the final stage of the PMS stellar evolution. Far-IR colours $\left[\lambda_{i}-\lambda_{j}\right]=m_{\lambda_{i}}-m_{\lambda_{j}}$ were calculated by using $m_{\lambda}=-2.5 \log F_{\lambda} / F_{\lambda_{0}}$, where $\lambda=12,25$ and $60 \mu \mathrm{m}$, and $F_{\lambda_{0}}$ is the IRAS calibration flux for zero magnitude. A source with [12-25] $=2.34$ and $[25-60]=2.82$ has a flat SED in the IRAS bands, while it would have a rising SED if the colours were greater than these limits. The results listed in Table 1 show that all of the objects have [25-60] indicating an increasing SED and half of them has [12-25] $>2.34$, meaning that these ones have a double-picked SED.

\subsection{Circumstellar Structure}

Models adopting two dust components have been suggested to explain the TT structure (Natta 1993, Calvet et al. 1994). In this case, the SED fit model assumes a system composed by a central star and the circumstellar disk, both surrounded by a spherical optically thin envelope. For the numerical simulations 
we considered the blackbody radiation with different temperature distribution for each component of the system: $T(r) \propto r^{-q}$, where $\mathrm{q}=0.75$ for the passive disk and $\mathrm{q}=0.4$ for the dust envelope. The best SED fit was obtained based on maximum likelihood statistics and $\chi^{2}$ tests for the goodness-of-fit (Hetem et al. 1994). Individual contribution in the SED could be evaluated in terms of emitted energy from the disk and the envelope, indicated by $L_{d}$ and $L_{e}$ in Table 1.

\section{Discussion}

By comparing the amount of lithium with the effective temperature we concluded that $46 \%$ of the studied sample could not be classified as WTT. The IR-excess was analysed in order to identify circumstellar structures showing evidences of protoplanetary disks. The larger amounts of circumstellar dust $\left(L_{d}+L_{e} \sim 0.3 L_{t o t}\right)$ and the shallower spectral indices $\left(a_{I R}>-0.5\right)$ can result from a disrupted disk: a double structure contributing to both near- and far-IR excess. The stars PDS30, 35, 145 and 156 were classified in this category. The other objects (PDS93, 109, 110, 111, 115, 116, 118 and 119) have $L_{d}+L_{e} \lesssim 0.2 L_{t o t}, a_{I R}<-1.0$, and IRAS colours typical of more evolved dust disks, showing a circumstellar structure more consistent with a $\beta$ Pic-like system.

It has been suggested that planet formation might be preceded by the disruption of the disk and the dispersal of the in the circumstellar dust of the young star. Considering the characteristics of the objects studied in this work, they are good candidates to be possibly producing protoplanetary disks.

\section{References}

André, P., \& Montmerle, T. 1994, ApJ, 420, 837

Briceño, C., Hartmann, L. W., Stauffer, J. R., Gagné, M., Stern, R. A., \& Caillault, J.-P. 1997, AJ, 113, 740

Calvet, N., Hartmann, L., Kenyon, S. J., \& Whitney, B. A. 1994, ApJ, 434, 330

Covino, E., Alcalá, J. M., Allain, S., Bouvier, J., Terranegra, L., \& Krautter, J. 1997, A\&A, 328, 187

Gregorio-Hetem, J., Lépine, J. R. D., Torres, C. A. O., Quast, G. R., \& de la Reza, R. 1992, AJ, 103, 549 (PDS I)

Hetem A., Gregorio-Hetem, J., Lepine, J.R.D. 1994, in Proceedings of the 10th IAP Astrophysics Meeting, Circumstellar Dust Disks and Planet Formation, ed. R. Ferlet \& A. Vidal-Madjar (Editions Frontiéres), 369

Martín, E. L. 1997, A\&A, 321, 492

Natta, A. 1993, ApJ, 412, 761

Torres, C. A. O., Quast, G. R., de la Reza, R., Lépine, J. R. D., \& Gregorio-Hetem, J. 1995, AJ, 109, 2146 (PDS II)

Wichmann, R., Covino, E., Alcalá, J. M., Krautter, J., Allain, S., \& Hauschildt, P. H. 1999, MNRAS, 307, 909

Wilking, B. A., Lada, C. J., \& Young, E. T. 1989, ApJ, 340, 823 